$S^{n}$, and $K$ is then a fibre bundle over $S^{n}$. Consequently we have the following commutative diagram of exact sequences

$$
\begin{aligned}
& \cdots \rightarrow \Pi_{k}\left(O_{n}\right) \rightarrow \Pi_{k}\left(O_{n+1}\right) \rightarrow \Pi_{k}\left(S^{n}\right) \rightarrow \Pi_{k-1}\left(O_{n}\right) \rightarrow \Pi_{k-1}\left(O_{n+1}\right) \rightarrow \cdots \\
& \begin{array}{lllll}
\downarrow i_{*} & \downarrow i_{*} & \downarrow j_{*} & \downarrow i_{*} & \downarrow i_{*}
\end{array} \\
& \cdots \rightarrow \Pi_{k}\left(K_{0}\right) \rightarrow \Pi_{k}(K) \rightarrow \Pi_{k}\left(S^{n}\right) \rightarrow \Pi_{k-1}\left(K_{0}\right) \rightarrow \Pi_{k-1}(K) \rightarrow \cdots
\end{aligned}
$$

where $j_{*}$ is the identity. It is easily seen that $\operatorname{Ker}\left(i_{*}: \Pi_{k}\left(O_{n+1}\right) \rightarrow \Pi_{k}(K)\right)$ is the image under $\Pi_{k}\left(O_{n}\right) \rightarrow \Pi_{k}\left(O_{n+1}\right)$ of $\operatorname{Ker}\left(i_{*}: \Pi_{k}\left(O_{n}\right) \rightarrow \Pi_{k}\left(K_{0}\right)\right)$. Since this last kernel is $O$ the theorem follows.

\title{
REFERENCES
}

1. D. Montgomery and L. Zippin, Topological transformation groups, New York, Interscience Publishers, 1955.

2. N. Steenrod, The topology of fibre bundles, Princeton, Princeton University Press, 1951.

UNiversity of Notre DAME

\section{A NOTE ON GAUSS' FIRST PROOF OF THE QUADRATIC RECIPROCITY THEOREM}

\section{CARLITZ}

We assume that the reader is familiar with Mathews' exposition $[1$, pp. 45-50] of the inductive proof of the reciprocity theorem. There are three main cases:

I. $p R q$,

II. $p N q, q \equiv 3(\bmod 4)$,

III. $p N q, q \equiv 1(\bmod 4)$.

In I we have $e^{2}-p=q^{f}$, in II we have $e^{2}+p=q^{f}$. In III we have first the lemma which asserts the existence of a prime $p^{\prime}<q$ such that $q N p^{\prime}$. This implies $p^{\prime} N q$, so that $p p^{\prime} R q$ and so $e^{2}-p p^{\prime}=q f$. In each of the cases I and II it is necessary to treat two sub-cases; in case III there are four sub-cases. Thus in all there are eight cases to consider.

We should like to point out in this note that it is possible to handle all cases simultaneously by introducing a little notation. To begin with, we define

Received by the editors September 22, 1959. 


$$
r=\left\{\begin{array}{cl}
1 & (\text { case I) } \\
-1 & \text { (case II) } \\
p^{\prime} & \text { (case III) }
\end{array}\right.
$$

Thus we have the single equation

$$
e^{2}-r p=q f,
$$

where $e$ is even and $<q, f$ is odd and $|f|<q$.

Next we put

$$
d=(f, r p), \quad f=d f^{\prime}, \quad e=d e^{\prime}, \quad r p=d d^{\prime},
$$

so that (1) becomes

$$
d e^{\prime 2}-d^{\prime}=q J^{\prime}
$$

moreover

$$
\left(f^{\prime}, d d^{\prime}\right)=\left(d, d^{\prime}\right)=\left(q, d d^{\prime}\right)=1 .
$$

From (2) we get $q f^{\prime} \equiv-d^{\prime}(\bmod 4)$, so that

$$
q+d^{\prime}+f^{\prime} \equiv 1(\bmod 4) \text {. }
$$

Now from (2) we also get

$$
\left(\frac{d d^{\prime}}{f^{\prime}}\right)=\left(\frac{q d f^{\prime}}{d^{\prime}}\right)=\left(\frac{-q d^{\prime} f^{\prime}}{d}\right)=1,
$$

which gives

$$
\left(\frac{q}{d d^{\prime}}\right)=\left(\frac{-d^{\prime}}{d}\right)\left(\frac{d}{d^{\prime}}\right)\left(\frac{f^{\prime}}{d d^{\prime}}\right) .
$$

We now apply the generalized reciprocity theorem:

$$
\left(\frac{m}{n}\right)\left(\frac{n}{m}\right)=-1^{(m-1)(n-1) / 4},
$$

where $m$ and $n$ are odd and relatively prime; also one of the numbers may be negative. The special cases $m$ or $n= \pm 1$ are included. Then we get, since $\left(d d^{\prime} / f^{\prime}\right)=1$,

$$
\left(\frac{q}{d d^{\prime}}\right)=(-1)^{\lambda}
$$

where 
I960] GAUSS' FIRST PROOF OF THE QUADRATIC RECIPROCITY THEOREM 565

$$
\lambda=\frac{1}{4}(d-1)\left(-d^{\prime}-1\right)+\frac{1}{4}\left(f^{\prime}-1\right)\left(d d^{\prime}-1\right) .
$$

Using (3) we find that

$$
\begin{aligned}
\lambda \equiv & \frac{1}{4}(d-1)\left(-d^{\prime}-1\right)-\frac{1}{4}\left(q+d^{\prime}\right)\left(d d^{\prime}-1\right) \\
\equiv & \frac{1}{4}(d-1)\left(-d^{\prime}-1\right)-\frac{1}{4}\left(d^{\prime}+1\right)\left(d+d^{\prime}-2\right) \\
& -\frac{1}{4}(q-1)\left(d d^{\prime}-1\right) \\
\equiv & -\frac{1}{4}\left(d^{\prime}+1\right)\left(2 d+d^{\prime}-3\right)-\frac{1}{4}(q-1)\left(d d^{\prime}-1\right) \\
\equiv & -\frac{1}{4}\left(d^{\prime 2}-1\right)-\frac{1}{2}\left(d^{\prime}+1\right)(d-1)-\frac{1}{4}(q-1)\left(d d^{\prime}-1\right) \\
\equiv & \frac{1}{4}(q-1)(r p-1)(\bmod 2) ;
\end{aligned}
$$

where at the last step we used $r p=d d^{\prime}$. Thus (4) becomes

$$
\left(\frac{q}{r p}\right)=(-1)^{(q-1)(r p-1) / 4}
$$

In cases I and II (5) is in obvious agreement with the reciprocity theorem; in III there is also agreement since we have $q N p^{\prime}$. Thus in III (5) reduces to

$$
\left(\frac{q}{p}\right)=-1
$$

which is the desired relation.

\section{REFERENCE}

1. G. B. Mathews, Theory of numbers, Cambridge, 1892.

DUKE UNIVERSITY 